\title{
Composite iterative schemes for maximal monotone operators in reflexive Banach spaces
}

\author{
Prasit Cholamjiak', Yeol Je Cho ${ }^{2^{*}}$ and Suthep Suantai ${ }^{3^{*}}$
}

\author{
* Correspondence: yjcho@gnu.ac.kr; \\ scmti005@chiangmai.ac.th \\ ${ }^{2}$ Department of Mathematics \\ Education and the RINS, \\ Gyeongsang National University, \\ Chinju 660-701, Korea \\ ${ }^{3}$ Department of Mathematics, \\ Faculty of Science, Chiang Mai \\ University, Chiang Mai 50200, \\ Thailand \\ Full list of author information is \\ available at the end of the article
}

\begin{abstract}
In this article, we introduce composite iterative schemes for finding a zero point of a finite family of maximal monotone operators in a reflexive Banach space. Then, we prove strong convergence theorems by using a shrinking projection method. Moreover, we also apply our results to a system of convex minimization problems in reflexive Banach spaces.

AMS Subject Classification: 47H09, 47H10
\end{abstract}

Keywords: Maximal monotone operator, Shrinking projection method, Proximal point algorithm, Bregman projection, Totally convex function, Legendre function

\section{Introduction}

Let $E$ be a real Banach space and $C$ a nonempty subset of $E$. Let $E^{*}$ be the dual space of $E$. We denote the value of $x^{*} \in E^{*}$ at $x 2 E$ by $\left\langle x^{*}, x\right\rangle$. Let $A: E \rightarrow 2^{E^{*}}$ be a setvalued mapping. We denote $\operatorname{dom} A$ by domain of $A$, that is, $\operatorname{dom} A=\{x \in E: A x \neq$ $\varnothing$ \}and also denote $G(A)$ by the graph of $A$, that is, $G(A)=f\left(x, x^{*}\right) \in E \times E^{*}: x^{*} \in$ $A x\}$. A set-valued mapping $A$ is said to be monotone if $\left\langle x^{*}-y^{*}, x-y\right\rangle \geq 0$ whenever $(x$, $\left.x^{*}\right) ;\left(y, y^{*}\right) \in G(A)$. It is said to be maximal monotone if its graph is not contained in the graph of any other monotone operator on $E$. It is known that if $A$ is maximal monotone, then the set $A^{-1}\left(0^{*}\right)=\left\{z \in E: 0^{*} \in A z\right\}$ is closed and convex.

The problem of finding zero points for maximal monotone operators plays an important role in optimizations. This is because it can be reduced to a convex minimization problem and a variational inequality problem. Many authors have studied the convergence of such problems in several settings, (see [1-6]). Initiated by Martinet [7], in a Hilbert space, Rockafellar [8] introduced the following iterative schemes:

$$
\left\{\begin{array}{l}
x_{1}=x \in E, \\
x_{n+1}=J_{\lambda_{n}} x_{n}, \quad \forall n \geq 1,
\end{array}\right.
$$

where $\left\{\lambda_{n}\right\} \subset(0, \infty)$ and $J_{\lambda}$ is the resolvent of $A$ defined by $J_{\lambda}=(I+\lambda A)^{-1}$ for all $\lambda$ $>0$, and $A$ is a maximal monotone operator on $E$. Such an algorithm is called the proximal point algorithm. He proved that the sequence $\left\{x_{n}\right\}$ generated by (1.1) converges weakly to an element in $A^{-1}(0)$ provided $\lim _{\inf _{n \rightarrow \infty}} \lambda_{n}>0$. Later, Kamimura and Takahashi [9] introduced the following iteration in a Hilbert space:

$$
x_{n+1}=\alpha_{n} x_{n}+\left(1-\alpha_{n}\right) J_{\lambda_{n}} x_{n}, \quad \forall n \geq 1,
$$

\section{SpringerOpen ${ }^{\circ}$}

(c) 2011 Cholamjiak et al; licensee Springer. This is an Open Access article distributed under the terms of the Creative Commons Attribution License (http://creativecommons.org/licenses/by/2.0), which permits unrestricted use, distribution, and reproduction in any medium, provided the original work is properly cited. 
where $\left\{\alpha_{n}\right\} \subset[0,1]$ and $\left\{\lambda_{n}\right\} \subset(0, \infty)$. The weak convergence theorems are also established in a Hilbert space under suitable conditions imposed on $\left\{\alpha_{n}\right\}$ and $\left\{\lambda_{n}\right\}$.

In 2005, Kohsaka and Takahashi [10] studied the above iteration process in a more general setting, reflexive Banach spaces. In fact, those authors proposed the following algorithm:

$$
x_{n+1}=\nabla f^{*}\left(\alpha_{n} \nabla f\left(x_{n}\right)+\left(1-\alpha_{n}\right) \nabla f\left(J_{\lambda_{n}} x_{n}\right)\right), \quad \forall n \geq 1,
$$

where $\left\{\alpha_{n}\right\} \subset[0,1],\left\{\lambda_{n}\right\} \subset(0, \infty), f: E \rightarrow \mathbb{R}$ is a Bregman function and $J_{\lambda}=(\nabla f+\lambda A)$

${ }^{-1} \nabla f$ for all $\lambda>0$. They also proved a weak convergence theorem of the proposed algorithm.

Very recently, in 2010, Reich and Sabach [11] proposed an algorithm for finding a zero point of maximal monotone operators $A_{i}: E \rightarrow 2^{E^{*}}(i=1,2, \ldots, N)$ in a general reflexive Banach space $E$ as follows:

$$
\left\{\begin{array}{l}
x_{0} \in E \\
y_{n}^{i}=\operatorname{Res}_{\lambda_{n}^{i} A_{i}}^{f}\left(x_{n}+e_{n}^{i}\right) \\
C_{n}^{i}=\left\{z \in E: D_{f}\left(z, y_{n}^{i}\right) \leq D_{f}\left(z, x_{n}+e_{n}^{i}\right)\right\} \\
C_{n}=\bigcap_{i=1}^{N} C_{n^{\prime}}^{i} \\
Q_{n}=\left\{z \in E:\left\langle\nabla f\left(x_{0}\right)-\nabla f\left(x_{n}\right), z-x_{n}\right\rangle \leq 0\right\}, \\
x_{n+1}=P_{C_{n} \cap Q_{n}}^{f}\left(x_{0}\right), \quad \forall n \geq 0,
\end{array}\right.
$$

where $\left\{\lambda_{n}^{i}\right\}_{i=1}^{N} \subset(0, \infty),\left\{e_{n}\right\}_{i=1}^{N}$ is an error sequence in $E$ with $e_{n}^{i} \rightarrow 0$ and $P_{K}^{f}$ the Bregman projection with respect to $f$ from $E$ onto a closed and convex subset $K$ of $E$. Those authors showed that the sequence $\left\{x_{n}\right\}$ defined by (1.4) converges strongly to a common element in $\bigcap_{i=1}^{N} A_{i}^{-1}\left(0^{*}\right)$ under some mild conditions.

Motivated by the previous ones, we first introduce a composite iterative scheme which is different from (1.4) for finding a zero point of maximal monotone operators $A_{i}: E \rightarrow 2^{E^{*}}(i=1,2, \ldots, N)$ in reflexive Banach spaces. Using the shrinking projection technique, introduced by Takahashi et al. [12], we then prove that a sequence generated by the proposed algorithm converges strongly to an element in $\bigcap_{i=1}^{N} A_{i}^{-1}\left(0^{*}\right)$ under some appropriate control conditions. Finally, we also apply our result to a system of convex minimization problems.

\section{Preliminaries and lemmas}

Let $E$ be a real reflexive Banach space with a norm $\|\cdot\|$ and $E^{*}$ be the dual space of $E$. Throughout this article, $f: E \rightarrow(-\infty,+\infty)$ is a proper, lower semi-continuous, and convex function, and the Fenchel conjugate of $f$ is the function $f^{*}: E^{*} \rightarrow(-\infty,+\infty]$ defined by

$$
f^{*}\left(x^{*}\right)=\sup \left\{\left\langle x^{*}, x\right\rangle-f(x): x \in E\right\} .
$$

We denote by $\operatorname{dom} f$ the domain of $f$, that is, the set $\{x \in E: f(x)<+\infty)$. For any $x \in$ int $\operatorname{dom} f$ and $y \in E$, the right-hand derivative of $f$ at $x$ in the direction $y$ is defined by

$$
f^{o}(x, y):=\lim _{t \rightarrow 0^{+}} \frac{f(x+t y)-f(x)}{t} .
$$


The function $f$ is said to be Gâteaux differentiable at $x \lim _{t \rightarrow 0^{+}} \frac{f(x+t y)-f(x)}{t}$ exists for any $y$. In this case, $f(x, y)$ coincides with $\nabla f(x)$, the value of the gradient $\nabla f$ of $f$ at $x$. The function $f$ is said to be Gâteaux differentiable if it is Gâteaux differentiable for any $x \in \operatorname{int} \operatorname{dom} f$. The function $f$ is said to be Fréchet differentiable at $x$ if this limit is attained uniformly in $\|y\|=1$. Finally, $f$ is said to be uniformly Fréchet differentiable on a subset $C$ of $E$ if the limit is attained uniformly for $x \in C$ and $\|y\|=1$.

Let $E$ be a reflexive Banach space. The Legendre function is defined from a general Banach space $E$ into $(-\infty,+\infty$ ] (see [13]). According to [13], the function $f$ is Legendre if and only if it satisfies the following conditions:

(L1) The interior of the domain of $f$ (denoted by int $\operatorname{dom} f$ ) is nonempty, $f$ * is Gâteaux differentiable on int $\operatorname{dom} f$, and $\operatorname{dom} \nabla f=\operatorname{int} \operatorname{dom} f$;

(L2) The interior of the domain $f^{*}$ (denoted by int $\operatorname{dom} f^{*}$ ) is nonempty, $f^{*}$ is Gâteaux differentiable on int $\operatorname{dom} f^{*}$, and $\operatorname{dom} \nabla f^{*}=\operatorname{int} \operatorname{dom} f^{*}$.

Since $E$ is reflexive, we always have $(\partial f)^{-1}=\partial f^{*}$ (see [14]). This fact, when combined with the conditions (L1) and (L2), implies the following equalities [15]:

$$
\begin{aligned}
\nabla f & =\left(\nabla f^{*}\right)^{-1}, \\
\operatorname{ran} \nabla f & =\operatorname{dom} \nabla f^{*}=\operatorname{int} \operatorname{dom} f^{*}, \\
\operatorname{ran} \nabla f^{*} & =\operatorname{dom} \nabla f=\operatorname{int} \operatorname{dom} f .
\end{aligned}
$$

Also, the conditions (L1) and (L2), in conjunction with [13], imply that the functions $f$ and $f^{*}$ are strictly convex on the interior of their respective domains. Several interesting examples of the Legendre functions are presented in $[13,16]$. Especially, the functions $\frac{1}{s}\|\cdot\|^{s}$ with $s \in(1, \infty)$ are Legendre, where the Banach space $E$ is smooth and strictly convex and, in particular, a Hilbert space. Throughout this article, we assume that the convex function $f: E \rightarrow(\infty,+\infty]$ is Legendre.

Lemma 2.1. [17] If $f: E \rightarrow \mathbb{R}$ is uniformly Fréchet differentiable and bounded on bounded subsets of $E$, then $\nabla f$ is uniformly continuous on bounded subsets of $E$ from the strong topology of $E$ to the strong topology of $E^{*}$.

Let $f: E \rightarrow(-\infty,+\infty]$ be a convex and Gâteaux differentiable function. The function $D_{f}: \operatorname{dom} f \times \operatorname{int} \operatorname{dom} f \rightarrow[0,+\infty)$ is defined as follows:

$$
D_{f}(y, x):=f(y)-f(x)-\langle\nabla f(x), y-x\rangle
$$

is called the Bregman distance with respect to $f$ [18].

Recall that the Bregman projection [19] of $x \in \operatorname{int} \operatorname{dom} f$ onto the nonempty, closed, and convex set $C \subset \operatorname{dom} f$ is necessarily the unique vector $P_{C}^{f}(x) \in C$ satisfying

$$
D_{f}\left(P_{C}^{f}(x), x\right)=\inf \left\{D_{f}(y, x): y \in C\right\}
$$

Let $f: E \rightarrow(-\infty,+\infty]$ be a convex and Gâteaux differentiable function. The function $f$ is said to be totally convex at $x \in \operatorname{int} \operatorname{dom} f$ if its modulus of total convexity at $x$, that is, the function $v_{f}$ : int $\operatorname{dom} f \times[0,+\infty) \rightarrow[0,+\infty]$ defined by

$$
v_{f}(x, t):=\inf \left\{D_{f}(y, x): y \in \operatorname{dom} f,\|y-x\|=t\right\}
$$

is positive, whenever $t>0$. The function $f$ is said to be totally convex when it is totally convex at every point $x \in \operatorname{int} \operatorname{dom} f$. In addition, the function $f$ is said to be 
totally convex on bounded sets if $v_{f}(B, t)$ is positive for any nonempty bounded subset $B$ of $E$ and $t>0$, where the modulus of total convexity of the function $f$ on the set $B$ is the function $v_{f}$ : int $\operatorname{dom} f \times[0,+\infty) \rightarrow[0,+\infty]$ defined by

$$
v_{f}(B, t):=\inf \left\{v_{f}(x, t): x \in B \cap \operatorname{dom} f\right\} .
$$

Let $C$ be a nonempty, closed, and convex subset of $E$. Let $f: E \rightarrow \mathbb{R}$ be a Gâteaux differentiable and totally convex function and let $x \in E$. It is known from [20] that $z=P_{C}^{f}(x)$ if and only if $\langle\nabla f(x)-\nabla f(z), y-z\rangle \leq 0$ for all $y \in C$. We also have

$$
D_{f}\left(y, P_{C}^{f}(x)\right)+D_{f}\left(P_{C}^{f}(x), x\right) \leq D_{f}(y, x), \quad \forall x \in E, y \in C .
$$

Recall that the function $f$ is said to be sequentially consistent [20] if, for any two sequences, $\left\{x_{n}\right\}$ and $\left\{y_{n}\right\}$, in $E$ such that the first is bounded:

$$
\lim _{n \rightarrow \infty} D_{f}\left(y_{n}, x_{n}\right)=0 \Rightarrow \lim _{n \rightarrow \infty}\left\|y_{n}-x_{n}\right\|=0 .
$$

The following lemmas were proved by Reich and Sabach [11].

Lemma 2.2. [11] Let $f: E \rightarrow \mathbb{R}$ be a Gâteaux differentiable and totally convex function. If $x_{0} \in E$ and the sequence $\left\{D_{f}\left(x_{n}, x_{0}\right)\right\}_{n=1}^{\infty}$ is bounded, then the sequence $\left\{x_{n}\right\}_{n=1}^{\infty}$ is also bounded.

We know that the resolvent of $A$, denoted by $\operatorname{Res}_{A}^{f}: E \rightarrow 2^{E}$, is defined as follows [21]:

$$
\operatorname{Res}_{A}^{f}(x)=(\nabla f+A)^{-1} \circ \nabla f(x) .
$$

It is known that $F\left(\operatorname{Res}_{A}^{f}\right)=A^{-1}\left(0^{*}\right)$, and $\operatorname{Res}_{A}^{f}$ is single-valued (see [21]). If $f$ is a Legendre function which is bounded, uniformly Fréchet differentiable on bounded, subsets of $E$, then $\hat{F}\left(\operatorname{Res}_{A}^{f}\right)=F\left(\operatorname{Res}_{A}^{f}\right)$ (see [22]). The Yosida approximation $A \lambda: E \rightarrow E$, $\lambda>0$, is also defined by

$$
A_{\lambda}(x)=\frac{1}{\lambda}\left(\nabla f(x)-\nabla f\left(\operatorname{Res}_{\lambda A}^{f}(x)\right)\right)
$$

for all $x \in E$. From Proposition 2.7 in [11], we know that $\left(\operatorname{Res}_{\lambda A}^{f}(x), A_{\lambda}(x)\right) \in G(A)$ and $0^{*} \in A x$ if and only if $0^{*} \in A_{\lambda} x$ for all $x \in E$ and $\lambda>0$.

Lemma 2.3. [11] Let $A: E \rightarrow 2^{E^{*}}$ be a maximal monotone operator such that $A^{-1}\left(0^{*}\right)$ $\neq \varnothing$. Then,

$$
D_{f}\left(p, \operatorname{Res}_{\lambda A}^{f}(x)\right)+D_{f}\left(\operatorname{Res}_{\lambda A}^{f}(x), x\right) \leq D_{f}(p, x)
$$

for all $\lambda>0, p \in A^{-1}\left(0^{*}\right)$ and $x \in E$.

\section{Strong convergence theorems}

Now, in this section, we prove our main results of this article.

Theorem 3.1. Let $E$ be a real reflexive Banach space and $f: E \rightarrow \mathbb{R}$ a Legendre function which is bounded, uniformly Fréchet differentiable and totally convex on bounded subsets of $E$. Let $A_{i}: E \rightarrow 2^{E^{*}}(i=1,2, \ldots, N)$ be maximal monotone operators such that $F:=\bigcap_{i=1}^{N} A_{i}^{-1}\left(0^{*}\right) \neq \emptyset$. Let $\left\{e_{n}\right\}_{n=1}^{\infty} \subset$ Ebe such that $\lim _{n \rightarrow \infty} e_{n}=0$. Define a sequence $\left\{x_{n}\right\}_{n=1}^{\infty}$ in $E$ as follows: 


$$
\left\{\begin{array}{l}
x_{1} \in E \\
C_{1}=E \\
y_{n}=\operatorname{Res}_{\lambda_{n}^{N} A_{N}}^{f} \circ \operatorname{Res}_{\lambda_{n}^{N-1} A_{N-1}}^{f} \circ \cdots \circ \operatorname{Res}_{\lambda_{n}^{1} A_{1}}^{f}\left(x_{n}+e_{n}\right), \\
C_{n+1}=\left\{z \in C_{n}: D_{f}\left(z, y_{n}\right) \leq D_{f}\left(z, x_{n}+e_{n}\right)\right\} \\
x_{n+1}=P_{C_{n+1}}^{f}\left(x_{1}\right), \quad \forall n \geq 1 .
\end{array}\right.
$$

If $\lim \inf _{n \rightarrow \infty} \lambda_{n}^{i}>0$ for each $i=1,2, \ldots, N$, then the sequence $\left\{x_{n}\right\}$ converges strongly to a point $P_{F}^{f}\left(x_{1}\right)$

Proof. We divide our proof into six steps as follows:

Step 1. $F \subset C_{n}$ for all $n \geq 1$.

Since $A_{i}^{-1}\left(0^{*}\right)$ is closed and convex for each $i=1,2, \ldots, N$, we get that $F:=\bigcap_{i=1}^{N} A_{i}^{-1}\left(0^{*}\right)$ is a nonempty, closed and convex subset of $E$. It is easy to see that $C_{n}$ is closed and convex for all $n \geq 1$. Indeed, for each $z \in C_{n}$, it follows that $D_{f}\left(z, y_{n}\right)$ $\leq D_{f}\left(z, x_{n}+e_{n}\right)$ is equivalent to

$$
\left\langle\nabla f\left(x_{n}+e_{n}\right)-\nabla f\left(y_{n}\right), z\right\rangle \leq f\left(y_{n}\right)-f\left(x_{n}+e_{n}\right)+\left\langle\nabla f\left(x_{n}+e_{n}\right), x_{n}+e_{n}\right\rangle-\left\langle\nabla f\left(y_{n}\right), y_{n}\right\rangle .
$$

This shows that $C_{n}$ is closed and convex for all $n \geq 1$. It is obvious that $F \subset C 1=E$. Now, suppose that $F \subset C_{k}$ for some $k \in \mathbb{N}$. For any $p \in F$, by Lemma 2.3, we have

$$
\begin{aligned}
D_{f}\left(p, y_{k}\right)= & D_{f}\left(p, \operatorname{Res}_{\lambda_{k}^{N} A_{N}}^{f} \circ \operatorname{Res}_{\lambda_{k}^{N-1} A_{N-1}}^{f} \circ \cdots \circ \operatorname{Res}_{\lambda_{k}^{1} A_{1}}^{f}\left(x_{k}+e_{k}\right)\right) \\
\leq & D_{f}\left(p, \operatorname{Res}_{\lambda_{k}^{N-1} A_{N-1}}^{f} \circ \operatorname{Res}_{\lambda_{k}^{N-2} A_{N-2}}^{f} \circ \cdots \circ \operatorname{Res}_{\lambda_{k}^{1} A_{1}}^{f}\left(x_{k}+e_{k}\right)\right) \\
& \cdots \\
\leq & D_{f}\left(p, \operatorname{Res}_{\lambda_{k}^{2} A_{2}}^{f} \circ \operatorname{Res}_{\lambda_{k}^{1} A_{1}}^{f}\left(x_{k}+e_{k}\right)\right) \\
\leq & D_{f}\left(p, \operatorname{Res}_{\lambda_{k}^{1} A_{1}}^{f}\left(x_{k}+e_{k}\right)\right) \\
\leq & D_{f}\left(p, x_{k}+e_{k}\right) .
\end{aligned}
$$

This implies that $F \subset C_{k+1}$. By induction, we can conclude that $F \subset C_{n}$ for all $n \geq 1$.

Step 2. $\lim _{n \rightarrow \infty} D_{f}\left(x_{n}, x_{0}\right)$ exists.

From $x_{n}=P_{C_{n}}^{f}\left(x_{1}\right)$ and $x_{n+1}=P_{C_{n+1}}^{f}\left(x_{1}\right) \in C_{n+1} \subset C_{n}$ we have

$$
D_{f}\left(x_{n}, x_{1}\right) \leq D_{f}\left(x_{n+1}, x_{1}\right), \quad \forall n \geq 1 .
$$

By (2.1), for any $p \in F \subset C_{n}$, we have

$$
D_{f}\left(x_{n}, x_{1}\right)=D_{f}\left(P_{C_{n}}^{f}\left(x_{1}\right), x_{1}\right) \leq D_{f}\left(p, x_{1}\right)-D_{f}\left(p, x_{n}\right) \leq D_{f}\left(p, x_{1}\right) .
$$

Combining (3.3) and (3.4), we know that $\lim _{n \rightarrow \infty} D_{f}\left(x_{n}, x_{1}\right)$ exists.

Step 3. $\lim _{n \rightarrow \infty}\left\|\nabla f\left(y_{n}\right)-\nabla f\left(x_{n}+e_{n}\right)\right\|=0$

Since $x_{m}=P_{C_{m}}^{f}\left(x_{1}\right) \in C_{m} \subset C_{n}$ for $m>n \geq 1$, by (2.1), it follows that

$$
\begin{aligned}
D_{f}\left(x_{m}, x_{n}\right)=D_{f}\left(x_{m}, P_{C_{n}}^{f}\left(x_{1}\right)\right) & \leq D_{f}\left(x_{m}, x_{1}\right)-D_{f}\left(P_{C_{n}}^{f}\left(x_{1}\right), x_{1}\right) \\
& =D_{f}\left(x_{m}, x_{1}\right)-D_{f}\left(x_{n}, x_{1}\right) .
\end{aligned}
$$

Letting $m, n \rightarrow \infty$, we have $D_{f}\left(x_{m}, x_{n}\right) \rightarrow 0$. Since $f$ is totally convex on bounded subsets of $E, f$ is sequentially consistent by Butnariu and Resmerita [20]. It follows that $\left\|x_{m}-x_{n}\right\| \rightarrow 0$ as $m, n \rightarrow \infty$. Therefore, $\left\{x_{n}\right\}$ is a Cauchy sequence. By the 
completeness of the space $E$, we can assume that $x_{n} \rightarrow q \in E$ as $n \rightarrow \infty$. In particular, we obtain

$$
\lim _{n \rightarrow \infty}|| x_{n+1}-x_{n} \|=0 .
$$

Since $e_{n} \rightarrow 0$, we also obtain

$$
\lim _{n \rightarrow \infty}\left\|x_{n+1}-\left(x_{n}+e_{n}\right)\right\|=0 \text {. }
$$

Since $x_{n+1}=P_{C_{n+1}}^{f}\left(x_{1}\right) \in C_{n+1}$

$$
\begin{aligned}
D_{f}\left(x_{n+1}, y_{n}\right) & \leq D_{f}\left(x_{n+1}, x_{n}+e_{n}\right) \\
& =f\left(x_{n+1}\right)-f\left(x_{n}+e_{n}\right)-\left\langle\nabla f\left(x_{n}+e_{n}\right), x_{n+1}-\left(x_{n}+e_{n}\right)\right\rangle .
\end{aligned}
$$

We know from [23] that, if $f$ is bounded on bounded subsets of $E$, then $\nabla f$ is also bounded on bounded subsets of $E$. Moreover, if $f$ is uniformly Fréchet differentiable on bounded subsets of $E$, then $f$ is uniformly continuous on bounded subsets of $E$ (see [24]). Using (3.5), we have

$$
\lim _{n \rightarrow \infty} D_{f}\left(x_{n+1}, y_{n}\right)=0 .
$$

Also, we have

$$
\lim _{n \rightarrow \infty}\left\|x_{n+1}-y_{n}\right\|=0
$$

and hence,

$$
\lim _{n \rightarrow \infty}\left\|y_{n}-x_{n}\right\|=0
$$

and, since $e_{n} \rightarrow 0$,

$$
\lim _{n \rightarrow \infty}\left\|y_{n}-\left(x_{n}+e_{n}\right)\right\|=0 .
$$

Since $f$ is uniformly Fréchet differentiable on bounded subsets of $E, \nabla f$ is norm-tonorm uniformly continuous on bounded subsets of $E$ by Lemma 2.1. Hence, we have

$$
\lim _{n \rightarrow \infty}\left\|\nabla f\left(y_{n}\right)-\nabla f\left(x_{n}+e_{n}\right)\right\|=0 .
$$

Step 4. $\lim _{n \rightarrow \infty}\left\|\nabla f\left(\Theta_{n}^{i}\left(x_{n}+e_{n}\right)\right)-\nabla f\left(\Theta_{n}^{i-1}\left(x_{n}+e_{n}\right)\right)\right\|=0 \forall i=1,2, \ldots, N$.

Denote $\Theta_{n}^{i}=\operatorname{Res}_{\lambda_{n}^{i} A_{i}}^{f} \circ \operatorname{Res}_{\lambda_{n}^{i-1} A_{i-1}}^{f} \circ \cdots \circ \operatorname{Res}_{\lambda_{n}^{1} A_{1}}^{f}$ for each $i \in\{1,2, \ldots, N\}$ and $\Theta_{n}^{0}=I$ for each $n \geq 1$. We note that $y_{n}=\Theta_{n}^{N}\left(x_{n}+e_{n}\right)$ for each $n \geq 1$. For any $p \in F$, by (3.2), it follows that

$$
\begin{aligned}
D_{f}\left(p, \Theta_{n}^{N-1}\left(x_{n}+e_{n}\right)\right) & \leq D_{f}\left(p, \Theta_{n}^{N-2}\left(x_{n}+e_{n}\right)\right) \\
& \leq D_{f}\left(p, \Theta_{n}^{N-3}\left(x_{n}+e_{n}\right)\right) \\
& \cdots \\
& \leq D_{f}\left(p,\left(x_{n}+e_{n}\right)\right) .
\end{aligned}
$$


Since $p \in A_{N}^{-1}\left(0^{*}\right)$, by Lemma 2.3 and (3.8), it follows that

$$
\begin{aligned}
D_{f} & \left(y_{n}, \Theta_{n}^{N-1}\left(x_{n}+e_{n}\right)\right) \\
\leq & D_{f}\left(p, \Theta_{n}^{N-1}\left(x_{n}+e_{n}\right)\right)-D_{f}\left(p, y_{n}\right) \\
\leq & D_{f}\left(p,\left(x_{n}+e_{n}\right)\right)-D_{f}\left(p, y_{n}\right) \\
= & f\left(y_{n}\right)-f\left(x_{n}+e_{n}\right)-\left\langle\nabla f\left(x_{n}+e_{n}\right), p-\left(x_{n}+e_{n}\right)\right\rangle+\left\langle\nabla f\left(y_{n}\right), p-y_{n}\right\rangle \\
= & f\left(y_{n}\right)-f\left(x_{n}+e_{n}\right)-\left\langle\nabla f\left(x_{n}+e_{n}\right), p-y_{n}\right\rangle \\
& +\left\langle\nabla f\left(x_{n}+e_{n}\right),\left(x_{n}+e_{n}\right)-y_{n}\right\rangle+\left\langle\nabla f\left(y_{n}\right), p-y_{n}\right\rangle \\
= & f\left(y_{n}\right)-f\left(x_{n}+e_{n}\right)+\left\langle\nabla f\left(y_{n}\right)-\nabla f\left(x_{n}+e_{n}\right), p-y_{n}\right\rangle \\
& +\left\langle\nabla f\left(x_{n}+e_{n}\right),\left(x_{n}+e_{n}\right)-y_{n}\right\rangle .
\end{aligned}
$$

From (3.6) and (3.7), we get that $\lim _{n \rightarrow \infty} D_{f}\left(y_{n}, \Theta_{n}^{N-1}\left(x_{n}+e_{n}\right)\right)=0$. Since $f$ is sequentially consistent,

$$
\lim _{n \rightarrow \infty}\left\|y_{n}-\Theta_{n}^{N-1}\left(x_{n}+e_{n}\right)\right\|=0
$$

Thus, from (3.6) and (3.9), it follows that

$$
\lim _{n \rightarrow \infty}\left\|\left(x_{n}+e_{n}\right)-\Theta_{n}^{N-1}\left(x_{n}+e_{n}\right)\right\|=0
$$

and hence,

$$
\lim _{n \rightarrow \infty}\left\|\nabla f\left(x_{n}+e_{n}\right)-\nabla f\left(\Theta_{n}^{N-1}\left(x_{n}+e_{n}\right)\right)\right\|=0 .
$$

Again, since $p \in A_{N}^{-1}\left(0^{*}\right)$, by Lemma 2.3 and (3.8), we know that

$$
\begin{aligned}
& D_{f}\left(\Theta_{n}^{N-1}\left(x_{n}+e_{n}\right), \Theta_{n}^{N-2}\left(x_{n}+e_{n}\right)\right) \\
& \quad \leq D_{f}\left(p, \Theta_{n}^{N-2}\left(x_{n}+e_{n}\right)\right)-D_{f}\left(p, \Theta_{n}^{N-1}\left(x_{n}+e_{n}\right)\right) \\
& \quad \leq D_{f}\left(p,\left(x_{n}+e_{n}\right)\right)-D_{f}\left(p, \Theta_{n}^{N-1}\left(x_{n}+e_{n}\right)\right) .
\end{aligned}
$$

From (3.10) and (3.11), we have

$$
\lim _{n \rightarrow \infty} D_{f}\left(\Theta_{n}^{N-1}\left(x_{n}+e_{n}\right), \Theta_{n}^{N-2}\left(x_{n}+e_{n}\right)\right)=0 .
$$

Since $f$ is sequentially consistent, it follows that

$$
\lim _{n \rightarrow \infty}\left\|\Theta_{n}^{N-1}\left(x_{n}+e_{n}\right)-\Theta_{n}^{N-2}\left(x_{n}+e_{n}\right)\right\|=0 .
$$

From (3.10) and (3.12), we have

$$
\lim _{n \rightarrow \infty}\left\|\left(x_{n}+e_{n}\right)-\Theta_{n}^{N-2}\left(x_{n}+e_{n}\right)\right\|=0,
$$

and hence,

$$
\lim _{n \rightarrow \infty}\left\|\nabla f\left(x_{n}+e_{n}\right)-\nabla f\left(\Theta_{n}^{N-2}\left(x_{n}+e_{n}\right)\right)\right\|=0 .
$$

In a similar way, we can show that

$$
\begin{gathered}
\lim _{n \rightarrow \infty}\left\|\Theta_{n}^{N-2}\left(x_{n}+e_{n}\right)-\Theta_{n}^{N-3}\left(x_{n}+e_{n}\right)\right\|=\cdots=\lim _{n \rightarrow \infty}\left\|\Theta_{n}^{1}\left(x_{n}+e_{n}\right)-\left(x_{n}+e_{n}\right)\right\|=0, \\
\lim _{n \rightarrow \infty}\left\|\left(x_{n}+e_{n}\right)-\Theta_{n}^{N-3}\left(x_{n}+e_{n}\right)\right\|=\cdots=\lim _{n \rightarrow \infty}\left\|\left(x_{n}+e_{n}\right)-\Theta_{n}^{1}\left(x_{n}+e_{n}\right)\right\|=0 \text { and }
\end{gathered}
$$




$$
\begin{aligned}
\lim _{n \rightarrow \infty} & \left\|\nabla f\left(x_{n}+e_{n}\right)-\nabla f\left(\Theta_{n}^{N-3}\left(x_{n}+e_{n}\right)\right)\right\| \\
= & \lim _{n \rightarrow \infty}\left\|\nabla f\left(x_{n}+e_{n}\right)-\nabla f\left(\Theta_{n}^{N-4}\left(x_{n}+e_{n}\right)\right)\right\| \\
& \cdots \\
= & \lim _{n \rightarrow \infty}\left\|\nabla f\left(x_{n}+e_{n}\right)-\nabla f\left(\Theta_{n}^{1}\left(x_{n}+e_{n}\right)\right)\right\| \\
= & 0 .
\end{aligned}
$$

Hence, we can conclude that

$$
\lim _{n \rightarrow \infty}\left\|\nabla f\left(\Theta_{n}^{i}\left(x_{n}+e_{n}\right)\right)-\nabla f\left(\Theta_{n}^{i-1}\left(x_{n}+e_{n}\right)\right)\right\|=0
$$

for each $i=1,2, \ldots, N$.

Step 5. $q \in \bigcap_{i=1}^{N} A_{i}^{-1}\left(0^{*}\right)$.

For each $i=1,2, \ldots, N$, we note that $\Theta_{n}^{i}\left(x_{n}+e_{n}\right)=\operatorname{Res}_{\lambda_{n}^{i} A_{i}}^{f} \Theta_{n}^{i-1}\left(x_{n}+e_{n}\right)$ and so

$$
\left\|A_{\lambda_{n}^{i}} \Theta_{n}^{i-1}\left(x_{n}+e_{n}\right)\right\|=\frac{1}{\lambda_{n}^{i}}\left\|\nabla f\left(\Theta_{n}^{i-1}\left(x_{n}+e_{n}\right)\right)-\nabla f\left(\Theta_{n}^{i}\left(x_{n}+e_{n}\right)\right)\right\| .
$$

From (3.13) and $\lim \inf _{n \rightarrow \infty} \lambda_{n}^{i}>0$, we have

$$
\lim _{n \rightarrow \infty}\left\|A_{\lambda_{n}^{i}} \Theta_{n}^{i-1}\left(x_{n}+e_{n}\right)\right\|=0 .
$$

We note that $\left(\Theta_{n}^{i}\left(x_{n}+e_{n}\right), A_{\lambda_{n}^{i}} \Theta_{n}^{i-1}\left(x_{n}+e_{n}\right)\right) \in G\left(A_{i}\right)$ for each $i=1,2, \ldots, N$. If $\left(w, w^{*}\right)$ $\in G(A i)$ for each $i=1,2, \ldots, N$, then it follows from the monotonicity of $A_{i}$ that

$$
\left\langle w^{*}-A_{\lambda_{n}^{i}} \Theta_{n}^{i-1}\left(x_{n}+e_{n}\right), w-\Theta_{n}^{i}\left(x_{n}+e_{n}\right)\right\rangle \geq 0 .
$$

Since $x_{n} \rightarrow q$ and $e_{n} \rightarrow 0, x_{n}+e_{n} \rightarrow q$. Therefore, $\Theta_{n}^{i}\left(x_{n}+e_{n}\right) \rightarrow q$ for each $i=1$, $2, \ldots, N$. Thus, from (3.14), we have

$$
\left\langle w^{*}, w-q\right\rangle \geq 0 \text {. }
$$

By the maximality of $A_{i}$, we have $q \in A_{i}^{-1}\left(0^{*}\right)$ for each $i=1,2, \ldots, N$. Hence, $q \in F:=\bigcap_{i=1}^{N} A_{i}^{-1}\left(0^{*}\right)$.

Step 6. $q=P_{F}^{f}\left(x_{1}\right)$.

From $x_{n}=P_{C_{n}}^{f}\left(x_{1}\right)$, we have

$$
\left\langle\nabla f\left(x_{1}\right)-\nabla f\left(x_{n}\right), x_{n}-z\right\rangle \geq 0, \quad \forall z \in C_{n} .
$$

Since $F \subset C_{n}$, we also have

$$
\left\langle\nabla f\left(x_{1}\right)-\nabla f\left(x_{n}\right), x_{n}-z\right\rangle \geq 0, \quad \forall z \in F .
$$

Letting $n \rightarrow \infty$ in (3.15), we obtain

$$
\left\langle\nabla f\left(x_{1}\right)-\nabla f(q), q-z\right\rangle \geq 0, \quad \forall z \in F .
$$

Hence, we have $q=P_{F}^{f}\left(x_{1}\right)$. This completes the proof.

As a direct consequence of Theorem 3.1, we also obtain the following result concerning a system of convex minimization problems in reflexive Banach spaces: 
Theorem 3.2. Let $E$ be a real reflexive Banach space and $f: E \rightarrow \mathbb{R}$ a Legendre function which is bounded, uniformly Fréchet differentiable, and totally convex on bounded subsets of $E$. Let $g_{i}: E \rightarrow(-\infty, \infty](i=1,2, \ldots, N)$ be proper lower semi-continuous convex functions such that $F:=\bigcap_{i=1}^{N}\left(\partial g_{i}^{-1}\right)(0) \neq \emptyset$. Let $\left\{e_{n}\right\}_{n=1}^{\infty} \subset$ Ebe a sequence in $E$ such that $\lim _{n \rightarrow \infty} e_{n}=0$. Define a sequence $\left\{x_{n}\right\}_{n=1}^{\infty}$ in $E$ as follows:

$$
\left\{\begin{array}{l}
x_{1} \in E, \\
C_{1}=E, \\
z_{n}^{1}=\arg \min _{\gamma \in E}\left\{g 1(\gamma)+\frac{1}{\lambda_{n}^{1}} D_{f}\left(y, x_{n}+e_{n}\right)\right\}, \\
\cdots \\
z_{n}^{N-1}=\arg \min _{y \in E}\left\{g N-1(\gamma)+\frac{1}{\lambda_{n}^{N-1}} D_{f}\left(y, z_{n}^{N-2}\right)\right\}, \\
y_{n}=\arg \min _{\gamma \in E}\left\{g N(y)+\frac{1}{\lambda_{n}^{N}} D_{f}\left(y, z_{n}^{N-1}\right)\right\}, \\
C_{n+1}=\left\{z \in C_{n}: D_{f}\left(z, y_{n}\right) \leq D_{f}\left(z, x_{n}+e_{n}\right)\right\}, \\
x_{n+1}=P_{C_{n+1}}^{f}\left(x_{1}\right), \quad \forall n \geq 1 .
\end{array}\right.
$$

If $\lim \inf _{n \rightarrow \infty} \lambda_{n}^{i}>0$ for each $i=1,2, \ldots, N$, then the sequence $\left\{x_{n}\right\}$ converges strongly to a point $P_{F}^{f}\left(x_{1}\right)$.

Proof. By Rockafellar's theorem [25,26], $\partial g_{i}$ are maximal monotone operators for each $i=1,2, \ldots, N$. Let $\lambda^{i}>0$ for each $i=1,2, \ldots, N$. Then $z^{i}=\operatorname{Res}_{\lambda^{i} g_{i}}^{f}(x)$ if and only if

$$
\begin{aligned}
0 & \in \partial g_{i}\left(z^{i}\right)+\frac{1}{\lambda^{i}}\left(\nabla f\left(z^{i}\right)-\nabla f(x)\right) \\
& =\partial\left(g_{i}+\frac{1}{\lambda^{i}}(f-\nabla f(x))\right)\left(z^{i}\right),
\end{aligned}
$$

which is equivalent to

$$
\begin{aligned}
z^{i} & =\arg \min _{y \in E}\left\{g_{i}(\gamma)+\frac{1}{\lambda^{i}}(f(y)-\langle y, \nabla f(x)\rangle)\right\} \\
& =\arg \min _{\gamma \in E}\left\{g_{i}(\gamma)+\frac{1}{\lambda^{i}} D_{f}(\gamma, x)\right\} .
\end{aligned}
$$

Using Theorem 3.1, we can complete the proof.

Remark 3.3. By means of the composite iterative scheme together with the shrinking projection method, we can construct the proximal point algorithms for finding a common element in the set $\bigcap_{i=1}^{N} A_{i}^{-1}\left(0^{*}\right)$. Moreover, our algorithm is different from that of Reich and Sabach [11] which is based on a finite intersection of sets.

Remark 3.4. Theorems 3.1 and 3.2 also hold in a uniformly convex and uniformly smooth Banach space with the generalized duality mapping. 


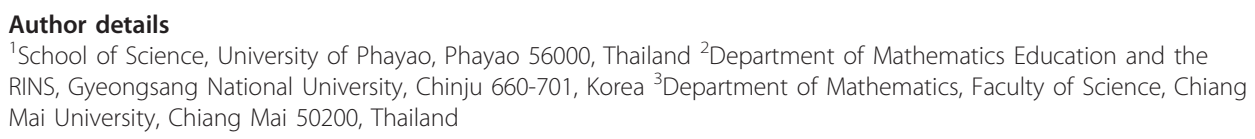

\section{Authors' contributions}

PC designed of the study, performed the nonlinear and convex analysis and also wrote the article. YJC participated in the design of the study, carried out the materials and helped to check the manuscript. SS conceived of the study, participated in its design and also helped to draft the manuscript. All authors read and approved the final manuscript.

\section{Competing interests}

The authors declare that they have no competing interests.

Received: 15 February 2011 Accepted: 22 June 2011 Published: 22 June 2011

\section{References}

1. Brézis H, Lions PL: Produits infinis de résolvantes. Israel J Math 1978, 29:329-345.

2. Burachik RS, lusem AN: A generalized proximal point algorithm for the variational inequality problem in a Hilbert space. SIAM J Optim 1998, 8:197-216.

3. Güler O: On the convergence of the proximal point algorithm for convex minimization. SIAM J Control Optim 1991, 29:403-419.

4. Passty GB: Ergodic convergence to a zero of the sum of monotone operators in Hilbert space. J Math Anal Appl 1979, 72:383-390.

5. Reich S, Sabach S: A projection method for solving nonlinear problems in reflexive Banach spaces. J Fixed Point Theory Appl.

6. Solodov MV, Svaiter BF: Forcing strong convergence of proximal point iterations in a Hilbert space. Math Program 2000, 87:189-202

7. Martinet B: Régularisation d'inéquations variationelles par approximations successives. Rev Francaise d'Informatique et de Recherche Opérationelle 1970, 4:154-159.

8. Rockafellar RT: Monotone operators and the proximal point algorithm. SIAM J Control Optim 1976, 14:877-898.

9. Kamimura S, Takahashi W: Approximating solutions of maximal monotone operators in Hilbert spaces. J Approx Theory 2000, 106:226-240.

10. Kohsaka F, Takahashi W: Proximal point algorithms with Bregman functions in Banach spaces. J Nonlinear Convex Anal 2005, 6:505-523.

11. Reich S, Sabach S: Two strong convergence theorems for a proximal method in reflexive Banach spaces. Numer Funct Anal Optim 2010, 31:22-44.

12. Takahashi W, Takeuchi $Y$, Kubota R: Strong convergence theorems by hybrid methods for families of nonexpansive mappings in Hilbert spaces. J Math Anal App/ 2008, 341:276-286.

13. Bauschke HH, Borwein JM, Combettes PL: Essential smoothness, essential strict convexity, and Legendre functions in Banach spaces. Commun Contemp Math 2001, 3:615-647.

14. Bonnans JF, Shapiro A: Perturbation Analysis of Optimization Problems. Springer Verlag, New York; 2000.

15. Reich S, Sabach S: Two strong convergence theorems for Bregman strongly nonexpansive operators in reflexive Banach spaces. Nonlinear Anal 2010, 73:122-135.

16. Bauschke HH, Borwein JM: Legendre functions and the method of random Bregman projections. J Convex Anal 1997, 4:27-67.

17. Reich S, Sabach S: A strong convergence theorem for a proximal-type algorithm in reflexive Banach spaces. $J$ Nonlinear Convex Anal 2009, 10:471-485.

18. Censor Y, Lent A: An iterative row-action method for interval convex programming. J Optim Theory App/ 1981, 34:321-353.

19. Bregman LM: The relaxation method for finding the common point of convex sets and its application to the solution of problems in convex programming. USSR Comput Math Math Phys 1967, 7:200-217.

20. Butnariu D, Resmerita E: Bregman distances, totally convex functions and a method for solving operator equations in Banach spaces. Abstr Appl Anal 2006, 2006:1-39, (Art ID 84919).

21. Bauschke HH, Borwein JM, Combettes PL: Bregman monotone optimization algorithms. SIAM J Control Optim 2003, 42:596-636.

22. Reich S, Sabach S: Existence and approximation of fixed points of Bregman firmly nonexpansive mappings in reflexive Banach spaces. In Fixed-Point Algorithms for Inverse Problems in Science and Engineering. Volume 49. Edited by: Bauschke HH et al. Springer, New York; 2011:301-316.

23. Butnariu D, lusem AN: Totally Convex Functions for Fixed Points Computation and Infinite Dimensional Optimization. Kluwer Academic Publishers, Dordrecht; 2000.

24. Ambrosetti A, Prodi G: A Primer of Nonlinear Analysis. Cambridge University Press, Cambridge; 1993.

25. Rockafellar RT: Characterization of the subdifferentials of convex functions. Pac J Math 1966, 17:497-510.

26. Rockafellar RT: On the maximal monotonicity of subdifferential mappings. Pac J Math 1970, 33:209-216.

doi:10.1186/1687-1812-2011-7

Cite this article as: Cholamjiak et al:: Composite iterative schemes for maximal monotone operators in reflexive Banach spaces. Fixed Point Theory and Applications 2011 2011:7. 